\title{
Schutz vor Pneumokokken-Erkrankungen
}

\section{Was taugt die Impfung für Senioren?}

\author{
Mit der seit 2006 empfohlenen generellen Impfung gegen \\ Pneumokokken für Kinder bis 24 Monate werden beeindruckende \\ Erfolge erzielt. In Bezug auf die einmalige Pneumokokkenimpfung \\ für Personen $a b 60$ Jahren sind dagegen noch viele Fragen offen.
}

\begin{abstract}
— Der bei der Impfung für Erwachsene eingesetzte Polysaccharidimpfstoff sorgt bei der Erstimpfung zuverlässig für hohe Antikörpertiter. Wie kann es sein, dass sich manche Geimpfte trotzdem eine Pneumokokkeninfektion zuziehen? „Das Problem ist, dass der Polysaccharidimpfstoff nur die B-Lymphozyten erreicht, aber nicht die für die lokale $\mathrm{Ab}$ wehr zuständigen T-Lymphozyten“, erläuterte Prof. Dr. Tobias Welte von der Klinik für Pneumologie der Medizinischen Hochschule Hannover bei den Praxistagen Pneumonie in Berlin. „Deshalb kann man durchaus erfolgreich geimpft sein und trotzdem eine Pneumokokkenpneumonie bekommen. Allerdings schützen die Antikörper vor der Bakteriämie und ihren Folgen", sagte Welte.
\end{abstract}

\section{Dünne Datenlage}

Der fehlende Schutz vor lokalen respiratorischen Pneumokokkeninfekten schränkt die Effektivität der Impfung ein. „Bei COPD-Patienten gibt es bis heute keinen Beleg dafür, dass sich durch die Impfung die Mortalitätsraten senken lassen", so Welte. Auch bei respiratorisch nicht vorbelasteten Personen über 60 Jahren ist die Datenlage dünn. Selbst eine jüngst publizierte und stark beachtete japanische Studie sieht Welte kritisch. Zwar war unter der Impfung bei den über 500 Bewohnern von Altenheimen (Durchschnittsalter 85 Jahre) die pneumokokkenassoziierte Mortalität und auch die Pneumonierate deutlich zurückgegangen. „Wenn man die Studie aber genauer ansieht, fällt auf, dass keine Aussagen zum Grippeimpfstatus gemacht wurden. Denkbar ist, dass die Betrof- fenen parallel gegen Grippe geimpft wurden", so Welte. Dadurch würde der Aussagewert auch dieser Studie erheblich relativiert.

\section{Empfehlung für Auffrischimpfung gestrichen}

Ein weiteres Problem ist die Auffrischungsimpfung. Bisher war es üblich nach fünf Jahren nachzuimpfen. „Diese Empfehlung ist jetzt sang- und klanglos aus den STIKO-Empfehlungen gestrichen worden", so Welte. Wiederholungsimpfungen werden jetzt nur noch bei bestimmten Indikationen empfohlen (Immundefekte mit T- und/oder B-ZellRestfunktion, chronische Nierenerkrankungen).

Über die Gründe kann man nur spekulieren. Die wahrscheinlichste Ursache ist, dass die Folgeimpfung mit dem Polysaccharidimpfstoff in der Regel keinen weiteren Antikörperanstieg auslöst. Laut Welte bedeutet das aber nicht zwangsläufig, dass die Auffrischimpfung wirkungslos ist, da es auch antikörperunabhängige Immunantworten gibt.

\section{Wirksamer Schutz vor invasiven Pneumokokkeninfektionen}

Eines dagegen ist mittlerweile gesichert: Kinder profitieren erheblich von der Impfung. Als geradezu sensationell bewertet Welte den Impferfolg des neuen konjugierten Impfstoffs, der für die Standardimpfung von Kleinkindern eingesetzt wird: „Bei geimpften Kindern sehen wir praktisch keine invasiven Pneumokokkenerkrankungen mehr. Früher gab es mehrere Hundert solcher tragischen Fälle. Die gute Durchimpfungsrate in Deutschland hat dazu geführt, dass sol- che schweren Erkrankungen praktisch auf null gefallen sind."

\section{Überraschende „Nebenwirkung“}

Die wirkliche Sensation ist jedoch ein Nebenbefund epidemiologischer Studien. Sie zeigen, dass die Impfung mit dem konjugierten Impfstoff nicht nur bei Kindern sehr gut wirkt. Auch die Erwachsenen im sozialen Umfeld profitieren erheblich von der Impfung der Kinder. „Durch die konsequente Impfung der Kinder halbierte sich die Rate der Pneumokokkenerkrankungen beim Erwachsenen", berichtete Welte. Offenbar stellt die okkulte Pneumokokkenbesiedlung der kindlichen Luftwege das Reservoir für viele Pneumokokkeninfektionen bei Erwachsenen dar.

\section{Konjugierte Vakzine stimuliert auch T-Lymphozyten}

Ursache für den unerwarteten sekundären Impferfolg ist die spezifischere Wirkung des konjugierten Impfstoffs. Er stimuliert nicht nur die für die Antikörper zuständigen B-Lymphozyten, sondern auch die für die lokale Abwehr verantwortlichen T-Lymphozyten. Durch die Impfung kommt es bei den Kindern quasi zur Pneumokokkeneradikation und damit zur Elimination eines signifikanten Keimreservoirs. Der Einsatz des konjugierten Impfstoffs ist theoretisch auch bei Erwachsenen problemlos möglich. Entsprechende Studien stehen allerdings noch aus.

\section{Dr. med. Horst Gross}

Quelle: 3. Pneumologische Praxistage, Berlin 30.4.-2.5.2010 (Veranstalter: Bundesverband der Pneumologen und Verband des Pneumologischen Assistenzpersonals in Deutschland) 